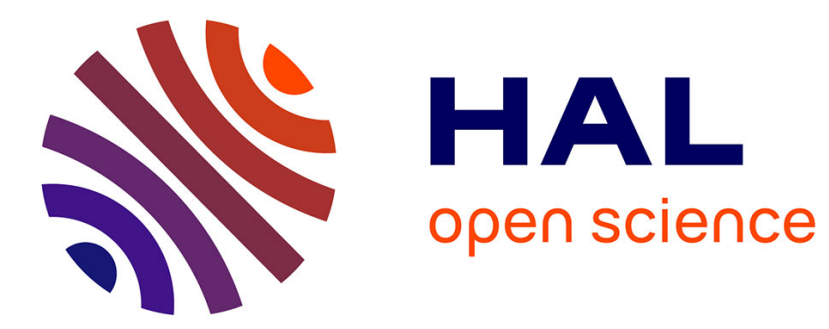

\title{
LE FLEXITARISME : ENTRE CARNIVORES SOCIAUX ET PRATIQUANTS DECOMPLEXES
}

Philippe Stefanini

\section{To cite this version:}

Philippe Stefanini. LE FLEXITARISME: ENTRE CARNIVORES SOCIAUX ET PRATIQUANTS

DECOMPLEXES. CORPS : Revue Interdisciplinaire, 2016. hal-02550595

\section{HAL Id: hal-02550595 \\ https://hal.science/hal-02550595}

Submitted on 22 Apr 2020

HAL is a multi-disciplinary open access archive for the deposit and dissemination of scientific research documents, whether they are published or not. The documents may come from teaching and research institutions in France or abroad, or from public or private research centers.
L'archive ouverte pluridisciplinaire HAL, est destinée au dépôt et à la diffusion de documents scientifiques de niveau recherche, publiés ou non, émanant des établissements d'enseignement et de recherche français ou étrangers, des laboratoires publics ou privés. 


\title{
LE FLEXITARISME : ENTRE CARNIVORES SOCIAUX ET PRATIQUANTS DECOMPLEXES
}

\author{
Philippe Stéfanini \\ Docteur en anthropologie biologique UMR7268 ADES/CNRS \\ philippe.stefanini@gmail.com
}

\section{Le flexitarisme est un omnivore à tendance semi-végétarienne. « Ce néologisme} est utilisé pour décrire une éco-pratique alimentaire singulière et un régime écologique, bon pour la santé »1.

Ce terme désigne également un processus évolutif mais régulier de régime alimentaire d'un individu, et ce selon les circonstances. Certaines comme le cas de notre étude, nous ont semblé pertinentes. Les individus qui pratiquent le flexitarisme sont nommés flexitariens « celui-ci ne peut-être considéré comme un végétarien, qui lui ne consomme pas de produits carnés. Mais il représente un nouveau type de consommateur qui choisit ce qu'il mange $»^{2}$. «Car pour eux, chaque fois les mêmes constatations positives reviennent sur le plan sanitaire des populations ayant adaptée ce mode de consommation $»^{3}$.

C'est pourquoi, nous avons entrepris d'étudier un terrain prédestiné à essaimer le flexitarisme: les agriculteurs bio, les producteurs de spiruline (aliment flexi ${ }^{4}$ par excellence) et les naturopathes.

En s'orientant vers ces éco-métiers certaines personnes ne recherchent pas uniquement une activité exemplaire, mais une vraie réflexion sur leur alimentation. Nous allons tenter de démontrer que ces individus au travers de leur métier et par leurs représentations, sont bien le terreau d'un véritable changement de leurs habitudes les guidant entre autres au flexitarisme. L'aliment au travers de l'alimentation a souvent été étudié du point de vue du consommateur, en accentuant sa haute charge affective ${ }^{5}$ ou symbolique ${ }^{6}$.

L'alimentation et la qualité des aliments nous imprègne par les représentations qu'ils véhiculent, ils permettent d'actualiser des souvenirs, des valeurs surtout quand celui-ci est d'origine animale : "l'exemple du bœuf en Chine, un interdit qui implique encore aujourd'hui un processus social, éthique et religieux en agriculture même après un siècle de révolution $»^{7}$.

\footnotetext{
${ }^{1}$ BITTMAN M., Définition : un flexitarien peut manger végétarien ou végétalien chez lui, mais manger des plats incluant de la viande lors d'occasions particulières comme aller au restaurant, lors de repas en famille ou chez des amis Un flexitarien est donc, par définition, un omnivore.

Ils s'imposent donc des contraintes alimentaires, plus souples que celles des végétariens, et ce pour différentes raisons comme des problèmes de santé, le souhait d'un traitement plus juste des animaux, ou des préoccupations environnementales, ou combiner toutes ces raisons.

En 2003, l'American Dialect Society a choisi le mot (anglais) flexitarian comme le néologisme le plus utile de l'année, le définissant comme « un végétarien qui mange occasionnellement de la viande », How to Cook Everything Vegetarian: Simple Meatless Recipes for Great Food Hardcover - October 15, 2007.

${ }^{2}$ BARBOULAT M., Le nouveau régime végétarien, Alpen, 2014, p. 5.

${ }^{3}$ GIGON F. et GALTIER, La méthode flexitarienne First, 2013, p. 35.

${ }^{4}$ LEVEBVRE C., Les carnivores infidèles, Cardinal, 2011, p 14.

${ }^{5}$ MOULIN Léo,. L'Europe à table, Paris, Elsevier. 1975, 204 p.

${ }^{6}$ FISCHLER Cl.,L'Homnivore-le goût, la cuisine et le corps, éd. Odile Jacob, Paris, 1990, 414 p.

${ }^{7}$ AUBIN F., « Vincent Gossaert, L'interdit du bœuf en Chine. Agriculture, éthique et sacrifice », Archives de sciences sociales des religions, 2007, p. 265.
} 
Pourtant, peu de travaux se penchent sur l'activité professionnelle qui les construit en symbole, si ce n'est les travaux sur leur éventuelle patrimonialisation ${ }^{8}$.

Comment certaines reconversions professionnelles peuvent faire basculer les habitudes alimentaires de certains et les mener au flexitarisme ? Leurs comportements face aux aliments carnés se transforment au fur et à mesure de leur réflexion, une nouvelle éthique apparaît et le engagent dans une autre démarche alimentaire.

«L'humain devient un avec l'animal qu'il ingère (le cadavre en putréfaction se perd dans les profondeurs sombres du corps humain) » ${ }^{9}$. Durand le qualifie de régime Mystique (ou antiphrastique) de l'imaginaire. Ici jouent à plein les principes d'analogie et de similitude. Le principe dynamique en œuvre est celui de la fusion.

Les pouvoirs donnés à certains aliments exceptionnels sont en général des symboles sociaux composés de croyances, de représentations et «parler de transformation, dès lors, implique une discussion des différents degrés de modification $\gg 10$.

Le recours à l'anthropologie permet de mettre en lumière ce phénomène de conversion vers le flexitarisme un mixage éthique entre un végétarisme dominant et un carnivore raisonné. Les sociétés contemporaines sont liée à la notion d'hétéro culture développé par Jean Poirier et qu'il définit comme la situation dans laquelle se trouve une société qui s'alimente à deux matrices culturelles considérées comme essentielles (et même probablement vitales) et antagonistes : la tradition et la modernité - autrement dit la continuité et la novation ${ }^{11}$. Aujourd'hui l'anthropologie peut-être considéré comme une discipline qui s'intéresse de plus en plus à la novation, aux transformations dans la modernité. Georges Balandier accentue ceci : «Les mouvements de la modernité font surgir du nouveau et de l'inédit, du mal connu et de l'inconnu au sein de notre propre société. Des domaines entiers nous y paraissent d'une certaine façon «étrangers » ou déconcertants ${ }^{12}$.

Nous intéressant notamment à cette dynamique de transformation des sociétés modernes, nous nous sommes penchés sur le flexitarisme en étudiant les capacités d'adaptation et de changement de certaines personnes devenant agriculteurs bio, spiruliniers et naturopathes.

De ce fait, ces nouveaux métiers vont au delà de l'aspect technique, ils engendrent de nouvelles représentations culturelles et sociales, de nouvelles rencontres, de nouveaux réseaux, de nouveaux comportements, venant ainsi façonner leur quotidien alimentaire et reconstruire parfois leur identité.

Car comme le souligne Claude Dubar «l'identité n'est jamais donnée, elle est toujours construite et (re) construite dans une incertitude plus ou moins grande et plus ou moins durable $»^{13}$.

Plus exactement, cette étude porte sur le processus de transformation qui s'opère chez l'individu qui passe d'une vision du monde à une autre grâce à leur nouvelle profession. Plus particulièrement, d'une agriculture et d'une médecine chimique à une démarche plus préventive et plus raisonnée.

\footnotetext{
${ }^{8}$ MICOUD André, 2003. « Ces bonnes vaches aux yeux si doux », Communication, n ${ }^{\circ} 74$ : 217-237. DUPRE Lucie, 2002. Du marron à la châtaigne. La relance d'un produit régional, Paris, CTHS.

${ }^{9}$ DURAND G.,Les structures anthropologiques de l'imaginaire, éd. Dunod, 1993, p. 125.

${ }^{10}$ BERGER P.et LUCKMANN T., La construction sociale de la réalité, éd. Méridiens Klincksieck, Paris, 1992.

11 POIRIER Jean, «Tradition et novation. De la "situation coloniale" à la "situation hétéroculturelle" », in Gabriel Gosselin (dir.), Les Nouveaux enjeux de l'anthropologie. Autour de Georges Balandier, Paris, L'Harmattan (coll. « Logiques sociales »), 1993, p. 75.

${ }^{12}$ BALANDIER G., «Regard Anthropologique sur la modernité et la post-modernité », in Corps, religion, société, présenté par F.LAPLANTINE, Presse Universitaire de Lyon, Lyon, p.117.

${ }^{13}$ DUBAR Claude, La socialisation : construction des identités sociales et professionnelles, Armand Colin, 2002, $255 \mathrm{p}$
} 
A partir d'observations participantes engagées sur plusieurs années, nous avons pu mettre en avant grâce à des entretiens avec de nombreux informateurs privilégiés, un processus de conversion lent mais solide vers un flexitarisme déterminé.

Nous avons tenté de mettre en relief les nombreuses représentations qu'ils se sont fabriquées sur la place des aliments carnés et de leurs dérivés dans leur alimentation quotidienne.

\section{HISTOIRE : ENTRE UN FLEXITARISME INVOLONTAIRE D'ADAPTATION A UN FLEXITARISME VOLONTAIRE RAISONNEE}

L'alimentation végétarienne ou omnivore des variantes permanentes au cours de l'histoire.

Par exemple, Pythagore est considéré par les occidentaux comme le premier végétarien de l'humanité qui ne vivait plus dans l'âge d'or, âge d'or où l'on était effectivement végétarien (mythologie hébraïque au travers de la bible jusqu'au déluge et philosophie grécoromaine «Cessez, mortels, de vous souiller de mets abominables! Vous avez les moissons ; vous avez les fruits dont le poids incline les rameaux vers la terre, les raisins suspendus à la vigne, les plantes savoureuses et celles dont le feu peut adoucir les sucs et amollir le tissu ; vous avez le lait des troupeaux, et le miel parfumé de thym ; la terre vous prodigue ses trésors, des mets innocents et purs, qui ne sont pas achetés par le meurtre et le sang. (...) ${ }^{14}$.

Depuis le moyen-âge, l'alimentation a évolué. Un courrier de l'explorateur Andrea Corsali à Guilano de Medicis faisait référence au statut alimentaire de Léonard de Vinci : «Certains infidèles nommés Hindous ne mangent rien qui contiennent du sang, et ne permettent aucune blessure faite à un être vivant, comme notre Léonard de Vinci ». ${ }^{15}$

En effet, l'alimentation de la plupart des gens de l'époque était très peu carnée : elle consistait en un bouillon de légumes (racines et herbes) auquel on ajoute, si on en a les moyens de la viande ou du poisson, dans laquelle on met des tranches de pain au moment de servir (on "trempe" la soupe). Le pain fournit l'apport principal en calories. A l'époque, blé sur les terres riches, sarrasin, méteil, épeautre. Pour les anciens grecs, l'homme ne peut manger le pain que s'il a été payé de sa peine, gagné à la sueur de son front. "C'est à la sueur de ton visage que tu mangeras ton pain"16 lit-on, dans la Genèse. D'autre part, depuis les temps les plus reculés, les céréales ont été, autour de la Méditerranée, les nourritures essentielles. Pour les anciens grecs, elles étaient "sitos", nourritures humaines, la religion chrétienne les a sanctifiées en faisant du pain un des éléments de l'eucharistie.

Autre exemple, la croyance autour du châtaignier : les châtaignes, nourritures ni vraiment humaines ni vraiment chrétiennes, ne pouvaient donc être que des nourritures subsidiaires et non pas des nourritures essentielles ${ }^{17}$.

Les XVIIe et XVIIIe siècles sont férus de progrès en matière d'agriculture (essais de nouvelles plantes et de nouvelles techniques agricoles) Ces nouveaux aliments développent de nouvelles habitudes alimentaires, on trouve désormais sur les tables françaises (maïs, pomme de terre, châtaigne, légumes secs, riz, vigne). Le sol est enrichi grâce à la culture du navet et des légumineuses fourragères on assiste à une première approche sur les engrais verts et l'émergence d'une première vision écologique.

\footnotetext{
${ }^{14}$ Pythagore ; $a$ et b Livre XV des Métamorphoses d'Ovide

${ }^{15}$ Lettre issue des traités de l'artiste, The Literary Works of Leonardo da Vinci, volume 2, p.617, par son traducteur Jean-Paul Richter.

${ }^{16}$ Genèse 3, verset 17-19.

${ }^{17}$ RICIARDI-BARTOLI F., Image de la châtaigne au travers l'histoire, 2009, pp. 105
} 
Le XIXème siècle est le siècle de la révolution agricole il amorce un nouveau tournant, il s'agit de s'intéresser à l'approche agronomique avec l'intention d'augmenter les rendements avec des variétés de céréales qui épuisent moins les sols. On assiste enfin à la naissance de l'individualisme agraire (mouvement des enclosures) qui transforme peu à peu l'agriculture de subsistance en une agriculture capitaliste. L'agriculture devient dés lors de plus en plus technique. L'alimentation devient plus abondante, plus accessible financièrement et plus variée.

Le XXème siècle se positionne avec une agriculture chimique, intensive définitivement moderne en oubliant des effets secondaires désastreux sur le plan biologique. Les habitudes alimentaires vont évoluer vers un apport de viande journalier plus important surtout après la deuxième guerre mondiale.

Aujourd'hui est revendiqué par les institutions une agriculture plus globale et plus respectueuse de l'environnement. Mais en parallèle, l'expérimentation de nouvelles espèces ou d'O.G.M. ${ }^{18}$ se développe à outrance.

L'aire de la biogénétique et de la biologie de synthèse au service de l'agriculture est en pleine expansion. La production animale est son impact néfaste sur la planète devient un enjeu majeur. Les nouvelles publications sur les effets pathologiques d'un excès de viande dans l'alimentation sont plus qu'alarmantes ${ }^{19}$.

$\mathrm{Au}$ XXI ème siècle nous avons internet et un accès rapide et simple au savoir. « Mais ne faudrait-il pas omettre que la connaissance passe par la raison et la raison passe par l'éthique et l'éthique passe par l'action $»^{20}$. Le flexitarisme n'est peut être pas un régime tendance mais peut-être les prémisses d'une prise de conscience individuelle. Certains métiers fédèrent et essaiment plus facilement cet état d'esprit.

\section{CONSOMMATION DE VIANDE}

En France, « la consommation individuelle moyenne de viandes des Français s'est établie à $87,8 \mathrm{~kg}$ en 2009, selon des chiffres diffusés par France Agri Mer issus de la France agricole MAP 2009. Elle a progressé de 10,1 kg par rapport à son niveau en 1970. La baisse par rapport au pic atteint en 1998 est toutefois de 6,7 kg par habitant. La progression de la population française permet à la consommation globale du pays d'afficher une stabilité depuis 1998, autour de 5,7 millions de tonnes équivalent carcasse. « La part des viandes blanches, particulièrement de volailles, s'accroît au détriment des viandes chevaline, ovine et bovine ». La consommation individuelle de viande bovine est passée de 30,1 kg en 1970 (39\%) à $25,4 \mathrm{~kg}$ en $2009(29 \%)$. Celle de porc a progressé de 30,7 kg en $1970(39 \%)$ à 34,3 kg en 2009 (39\%), et celle de volailles de $12,2 \mathrm{~kg}(16 \%)$ à $24,2 \mathrm{~kg}(28 \%) » 21$.

«En Europe, la consommation de viandes par habitant va de $34 \mathrm{~kg}$ par an en Roumanie et $49 \mathrm{~kg}$ par an en Bulgarie, à $104 \mathrm{~kg}$ en Espagne et $135 \mathrm{~kg}$ pour Chypre » 22 .

Nos publics cibles ont dans l'ensemble pour ambition de transformer leur vie quotidienne dans un esprit plus éthique. Ils veulent être des promoteurs d'un changement de vie alliant la qualité des productions agricoles et de l'alimentation. La baisse de l'alimentation carnée en France est pour eux un enjeu majeur de leur démarche professionnelle qui est de fait dans un esprit environnementaliste.

\footnotetext{
${ }^{18}$ Organisme Génétiquement Modifié

${ }^{19}$ Etude de juin 2005, des Dr Elio Riboli et Teresa Norat, in JNCI -Journal of the National Cancer Institut

${ }^{20}$ SPINOZA B., Spinoza : æuvvre complète, Arvensas, 2014, pp 1425.

${ }^{21}$ Source : CRÉDOC, Enquête CCAF 2010

${ }^{22}$ Les cahiers de France AGRI/MER, Chiffres Clefs, 2010.
} 
Ils semblent passer, d'un statut de consommateurs candides à des bienfaiteurs de la nature et de leur corps, d'une reconversion professionnelle à une conversion éthique.

Ainsi, à partir de l'investigation du terrain, est malgré une connaissance en nutrition et du langage vernaculaire est venu à moi des constats de singularités sociales insoupçonnées.

La dimension professionnelle des identités acquiert une importance particulière.

E. Hugues ${ }^{23}$ met l'accent sur le fait essentiel que le «monde vécu au travail » ne pouvait se réduire à une simple transaction économique (l'usage de la force dans le travail) : il met en jeu la personnalité individuelle et l'identité sociale du sujet, il cristallise ses espoirs et son image de soi, il engage sa définition et sa reconnaissance sociale. De ce fait, leur métier conditionne la construction d'identités sociales : parce qu'elle connaisse comme dans mon cas d'étude des $«$ mutations alimentaires ${ }^{24}$. Certaines « démarches passions » comme le flexitarisme induisent des transformations identitaires délicates surtout quand la formation intervient bien au delà de la période scolaire. Nous avons remarqué lors de nos entretiens que nos informateurs devenus flexitariens après leur formation essayent d'éviter l'asocialisation en restant carnivore. Leur nouveau métier leur a inculpé de nouveaux modes de penser, de sentir, d'agir. Une des conséquences du flexitarisme est de rendre stables des dispositions du comportement ainsi acquises. Cette intériorisation des normes et valeurs a également pour fonction de rendre siennes de nouvelles règles sociales, qui sont par définition extérieures à l'individu et d'augmenter la solidarité entre les membres du groupe ${ }^{25}$. Cependant, ce concept est précisé afin de décrire l'acquisition d'une nouvelle pratique professionnelle influençant une nouvelle façon de s'alimenter, la socialisation représente les études portant sur les différents types d'apprentissages auxquels sont soumis nos informateurs et comment ceux-ci construisent tout au long de leur parcours une vision nouvelle de la nutrition. Cette socialisation professionnelle apparaît donc très complexe aussi bien du point de vue «structurel » de leur insertion dans leurs nouveaux savoirs que de point de vue «biographique » de leur construction d'une compétence nouvellement reconnue. Si nous évoquons la notion de socialisation par mes publics cibles (ayant pour objet l'amélioration d'un état ressenti et jugé déficient), c'est pour mieux appréhender l'apprentissage de ces nouvelles formations en tant qu'enseignement structuré. Dans tout processus social et notamment dans la socialisation, sont mis en mouvement des sujets agissant et réagissant. Il est nécessaire d'analyser la dynamique qui s'opère chez mes interlocuteurs, lors des processus d'apprentissage empirique auxquels ils ont pu être soumis et comment ces nouvelles connaissances transcendent ce processus.

Pour mes informateurs, leur transformation décomplexée a pu débuter quand ils ont commencé à changer leur vision des aliments, leur apprentissage s'est établi sur le schéma Méadien ${ }^{26}$ plus précisément la problématique des savoirs élaborée par le courant phénoménologique ${ }^{27}$.

La socialisation se définit avant tout par l'immersion des individus sans ce qu'il appelle « monde vécu » qui est tout à la fois un « univers symbolique et culturel » et un « savoir sur ce monde ». L'enfant absorbe le monde social dans lequel il vit « non pas comme un univers possible parmi d'autres mais comme le monde. Cette socialisation primaire se fait avant tout par ses éducateurs et elle s'ensuit par une socialisation secondaire venant entre autre enrichir

\footnotetext{
${ }^{23}$ HUGUES E., Le regard sociologique, Essais choisis, Textes rassemblés et présentés par J. M. Chapoulie, Paris, Ed de l'E.H.E.S.S. .

${ }^{24}$ CHARLOT B., éd. GLASMAN D. , Les jeunes, l'insertion et l'emploi, Paris, PUF, 1998. p. 27.

${ }^{25}$ CHERKAOUI M., « socialisation », Dictionnaire, Larousse, 1990, p.181.

${ }^{26}$ MEAD K. H.(1933), Mind, Self and Society, trad. L'esprit, le soi et la société, présentation de J. Caseneuve, Paris, PUF, 1963.

${ }^{27}$ Mené notamment par SCHUTZ A., The Phenomenology of the Social World Evasion, North Western University Press, 1967.
} 
le capital de connaissance de l'individu avec des savoirs spécifiques, professionnels. C'est ainsi que seront intériorisés, valeurs, normes, codes symboliques constituant une culture personnalisée avec notamment «intériorisation de sous-mondes institutionnels spécialisés ${ }^{28}$. Ces savoirs spécialisés seront appelés savoirs professionnels qui constituent des savoirs d'un genre nouveau. Quelle est la part des effets de la socialisation dans l'explication du binôme conversion éthique et flexitarisme ? Avant tous changements majeurs, l'hybridation est une phase intermédiaire, prépondérante de notre début de siècle par exemple entre les individus, les voitures, les régimes alimentaires le monde devient de plus en plus flexible et adaptable au changement environnementaux. Le flexitarisme ne déroge pas à la règle l'éthique structure cette nouvelle culture alimentaire.

Notre étude a permis de montrer l'évolution des représentations et des comportements de nos informateurs après leur installation: comment ils revisitent la notion d'aliments, en développant leur quête de santé, de vérité. En mangeant mieux, ils pensent également devenir des exemples avant-gardistes.

Dans cet apprentissage graduel, une évolution de leurs savoirs, de leurs pratiques et de leur croyance a été observée. La pensée d'une éthique écologique dégagée par l'alimentation est revisitée par ces carnivores sociaux Elle devient comme l'écrit Gaston Bachelard «l'imago » de leur énergie. Autrement dit, la matière est notre miroir énergétique : c'est un miroir qui focalise nos puissances en les illuminant de joies imaginaires» (Bachelard, 2004).

\section{RESULTATS DE NOTRE ETUDE CONCERNANT LE FLEXITARISME}

Résultats finals de notre étude sur 4 ans portant sur l'entretien individuel de 150 stagiaires devenus professionnels :

- $70 \%$ sont devenus flexitariens, s'alimentent majoritairement en bio et promotionnent leur nouveau statut,

- $20 \%$ s'alimentent en bio pour les denrées de base (laitages, œufs, céréales,...)

$-10 \%$ n'ont rien fait.

\section{CONCLUSION}

Comme il faut bien vivre ensemble, on voit parfois se développer des éthiques et des comportements particuliers comme le flexitarisme. «Ceux-ci traduisant ce "sentiment d'appartenance " propre aux tribus postmodernes » (Maffesoli, 2007).

A l'écoute de tous nos publics cibles, il apparait, malgré de fortes individualités et des itinéraires différents, une étonnante permanence de tout un système de représentations. Des agriculteurs aux naturopathes divers modèles de perception du monde sont engendrés ils servent tous à en justifier une certaine pratique, visant à déboucher sur une éthique environnementale et pour une majorité sur le flexitarisme. Celle-ci s'est structurée comme un « phénomène social total » (Mauss, 1994), ce qui a eu pour effet de transformer leur propre vision du monde. Dans ce travail, nous avons essayé d'analyser ce phénomène afin de contribuer au débat lié au processus de conversion de certains groupes sociaux.

Au-delà de choisir nos groupes cibles, producteurs de spiruline, agriculteurs bio, naturopathes et stagiaires en formation, le premier objectif a été de bien analyser et définir notre objet

\footnotetext{
${ }^{28}$ BERGER P. \& LUCKMANN T., La construction sociale de la réalité, éd. Méridiens klincksieck, Paris, 1992, P.214.
} 
d'étude selon une approche pluridisciplinaire aussi fiable que possible. Le second objectif a été de mettre en évidence un mode alimentaire qui se développe de plus en plus dans en occident sous l'influence de certaines minorités. De ce fait, nous avons pu étudier l'évolution de leurs représentations, de leurs pratiques, de leurs comportements, de leurs croyances et de leur essaimage consciente ou non inconsciente sur la société. Nous avons essayé de mettre en évidence une démarche nouvelle selon laquelle l'alimentation et l'aliment peuvent agir en termes de représentations sur celui qui cultive ou qui éduque. Afin de mener à bien cette étude, nous avons conduit notre recherche, de l'individu aux effets sur le groupe, afin de montrer qu'il s'agit bien pour ces personnes d'une réelle reconstruction alimentaire.

\section{Une réalité concrète porteuse de sens}

A travers les notions de qualités nutritionnelles, d'aliments bio, d'alimentation carnée, d'éducation à la santé, leur nouvelle tendance nutritionnelle a redonné du sens à leur vie. Ainsi, aujourd'hui, ils semblent avoir atteint un épanouissement et une maturité tant recherchés, ils sont porteurs de nouvelles valeurs et une majorité sont devenus flexitariens ce nouveau statut est assumé et revendiqué.

Ils ont enfin trouvé un métier et un mode de vie valorisant qui répond à leurs attentes et à leurs espérances.

\section{Un itinéraire, une rencontre, de nouvelles valeurs}

Durant cette étude, nous avons voulu mettre en avant les motivations qui ont poussé ces individus à transformer leurs habitudes les plus profondes. Pour Weber, comprendre et expliquer un comportement ou une valeur chez l'acteur suppose de retrouver ses causes, ses motivations. Il semblerait que ces représentations aient été l'objet de transformations ou de développement au cours d'une évolution adaptative à un nouveau métier. Car l'homme serait donc capable de construire et de « réinterpréter progressivement un nouveau système culturel pouvant aboutir à des innovations dans les valeurs morales » (Weber, 1992). Au-delà de se construire un monde meilleur, ces nouvelles représentations lui permettent de communiquer sur de nouveaux schémas de vie, l'ouvrant sur d'autres symboles et sur d'autres personnes. « L'aliment doit non seulement être un objet nutritionnel, écrivait Jean Trémolières, mais aussi faire plaisir et posséder une signification symbolique » (Trémolières, 1984).

Devenir flexitarien n'est pas un acte anodin, il s'inscrit dans une démarche portée par des convictions rationnelles et irrationnelles car, comme nous le montre Saadi Lahlou, « la représentation de manger (...) renvoie à des objets (les aliments) mais aussi à des émotions, des valeurs, et des rituels (inscrits par exemple dans une chronologie et dans des lieux précis) » (Lalhou, 1998). Mais quand l'acte de manger de la viande s'associe à la santé de l'homme et de la planète comme dans le cas de notre étude, nos informateurs se sont engagés dans la valeur de l'exemple.

L'expérimentation de leur nouveau métier aura validé le monde qu'ils désiraient. Une nouvelle réalité aura été construite tout au long de leur apprentissage. Ils ont effacé une partie de leurs anciennes représentations alimentaires car elles n'étaient plus opératoires. Au-delà de leur conversion, nos informateurs recherchent l'harmonie entre leur identité et leur vision intime du monde.

Au-delà de l'adoption d'une nouvelle pratique aimentaire, ces individus ont vécu un vrai changement de leur système de pensée en fusionnant dans leur quotidien, mode de vie, activité professionnelle et éthique alimentaire. 


\section{Retisser des liens avec la nature et le sacré dans une dimension symbolique}

La plupart, pensent qu'au fil du temps, ils se sont éloignés de la nature. Ils prétendent que la tentation est grande de polluer dans cette agriculture car elle encourage à produire «toujours plus ». En fait, ils sont persuadés d'avoir sombré dans une civilisation de plus en plus matérialiste où la seule recherche est celle du gain à n'importe quel prix.

La rencontre avec le flexitarisme semble leur avoir ouvert un espace oublié: celui du sacré.

Dans cette démarche, ils espèrent se transcender en réalisant un subtil mélange, alimentation carnée et végétarienne. Cette nouvelle culture en émergence implique un perpétuel bricolage nutritionnel qui a pour objectif de valider les qualités exceptionnelles de cet aliment singulier qui garantie des apports protéiniques de grande qualité. En réactualisant leur mode alimentaire, ils souhaitent peut-être inconsciemment réintroduire le sacré dans son existence et dans la société qui voit perdre tous ses points de repères par un trop grand désir de pragmatisme et de rationalité (Balandier, 1988).

\section{Action du groupe sur la société}

L'effet du groupe, surtout par la valeur de l'exemple a impulsé une dynamique du changement.

$\mathrm{Au}$ fur et à mesure, ils ont partagé de nombreux repas en toute conscience et une identité commune est née entraînant un sentiment d'appartenance.

Leur identité se restructure, un esprit de liberté se conscientise. Dès lors, ils réinterprètent individuellement les choses et ils intègrent progressivement de nouvelles représentations. Cette perception individuelle puis collective a été engendrée comme un phénomène de contagion entraînant un déroulement combinatoire se déplaçant des anciennes représentations vers de nouvelles représentations. Ce processus de conversion prenant pour assise philosophique, l'éthique alimentaire. A partir de ce moment, ils commencent à se singulariser et à entrevoir la possibilité d'un monde meilleur et commence a impacter tout le reste de la société à l'instar du grand chef français Alain Ducasse qui a lancé plusieurs restaurants dans un esprit entièrement flexitariens ${ }^{29}$.

\section{Une singularité postmoderne}

Ils ont préféré mener une démarche introspective, restructurante qui s'appuie sur de nouvelles valeurs. Ils se singularisent au profit de leur nouvelle alimentation En se détournant de leur ancienne image négative, ils se sentent protégés, leur nouveau métier les régénère et les épanouit.

De par leur apprentissage, ils ont tenté d'échapper à une vie de soumission dénudée de sens. Ils puisent dans leur nouvelle activité et la partagent avec leur nouveau réseau pour se rassurer et se sécuriser. Au-delà de leur vie professionnelle, leur vie privée prend dorénavant plus d'ampleur, ils se sentent comme portés par une éthique alimentaire qu'ils définissent de « raisonnée ». Leur nouveau métier a changé les paramètres de leur chemin de vie, en produisant, en s'alimentant autrement ils sont persuadés d'agir sur leur environnement, sur eux et sur les gens. Aujourd'hui, l'alimentation comme le système de santé sont de plus en plus remis en cause par les consommateurs, les patients qui semblent affectés les différents

\footnotetext{
${ }^{29}$ ConsoGlobe, 2 octobre 2014, Restaurant à tendance végétarienne d'Alain DUCASSE le Plaza Athénée.
} 
scandales de ces dernières années (cultures d'O.G.M., bœufs aux hormones, vin au vinaigre, faux labels, présence de métaux lourds dans les aliments, propagation de l'encéphalopathie spongiforme bovine (E.S.B.), Médiator ...). Toutes ces controverses amènent le doute et la critique sur ces professions, qui par conséquent amène les professionnels à s'interroger sur leur devenir. Cette singularité sociale qui s'établit autour de métiers résolument moderne est peut-être une ouverture vers de nouveaux modèles qui peuvent interroger les dysfonctionnements d'une alimentation en perte de repères.

En étant flexitarien, ils se sentent désormais plus libres qu'avant et se positionnent aisément dans leurs actes alimentaires.

\section{Discussion, perspectives et applications}

Cette étude a montré que leur nouveau métier a été le terreau d'une véritable conversion alimentaire décomplexée dénommée flexitarisme.». Mais à l'écoute de notre terrain, nous avons pu constater que ce nouveau régime avait touché à leur identité de consommateur, car il prenait racine dans leurs réflexions antérieures et dans leur activité professionnelle. Pour finir, afin d'ouvrir, au-delà de l'objet central de ce travail, il ne s'agit pas tant de conclure que d'esquisser d'autres espaces de questionnement.

Celui que nous souhaitons développer est à double entrée, mais renvoie à une problématique commune: en quoi le flexitarisme que ces éco-métiers véhicule, nous conduit-il à reconsidérer les pratiques et les discours de l'Homme postmoderne ? Comment, traitant d'un objet à mi-chemin entre l'origine des pratiques alimentaires, la croyance et le futur de l'homme, prendre acte de l'enjeu du corps écologique? Est-ce que par ce type de mouvement singulier et contestataire, les mouvements néosociaux comme le flexitarisme peuvent devenir opérants?

Aujourd'hui, comme nous l'avons vu, cette nouvelle tendance permet de mettre en place un nouveau débat social autour de la place de l'aliment carné dans nos habitudes alimentaires par exemple la grande marque Bjorg semble déjà prendre les choses en main ${ }^{30}$.

Sur quels arguments ce débat se construit-il ?

- Le flexitarisme propose une alternative alimentaire qui bouleverse l'existence de celui qui le pratique et l'essaime en même temps.

Les résultats de cette étude méritent d'être enrichis car le phénomène étudié s'implique prioritairement sur un niveau de conversion qui est celui de l'éthique alimentaire. Mais un autre niveau pourrait être mis en perspective et approfondi: la conversion d'une partie des espaces de production animales au profit de productions végétales riches en protéines et protectrice de l'environnement.

\footnotetext{
${ }^{30}$ Scoop.it, 10 mars 2015, Bjorg déclare :le flexitarisme est la tendance alimentaire qui monte.
} 